\title{
Boolean logic device done with DFB laser diode
}

\author{
A. Hurtado, A.P. Gonzalez-Marcos, J.A. Martin-Pereda \\ E.T.S. Ingenieros de Telecomunicación. Universidad Politécnica de Madrid \\ Ciudad Universitaria. 28040 Madrid. Spain
}

\begin{abstract}
We present simulation results on how power output-input characteristic bistability in Distributed FeedBack -DFB semiconductor laser diode SLA can be employed to implemented Boolean logic device. Two configurations of DFB Laser diode under external optical injection, either in the transmission or in the reflective mode of operation, is used to implement different Optical Logic Cells (OLCs), called the Q- and the P-Device OLCs. The external optical injection correspond to two inputs data plus a cw control signal that allows to choose the Boolean logic function to be implement. DFB laser diode parameters are choosing to obtain an output-input characteristic with the values desired. The desired values are mainly the on-off contrast and switching power, conforming shape of hysteretic cycle. Two DFB lasers in cascade, one working in transmission operation and the other one in reflective operation, allows designing an inputoutput characteristic based on the same respond of a self-electrooptic effect device is obtained. Input power for a bit" 1 " is $35 \mu \mathrm{W}(70 \mu \mathrm{W})$ and a bit " 0 " is zero for all the Boolean function to be execute. Device control signal range to choose the logic function is $0-140 \mu \mathrm{W}(280 \mu \mathrm{W})$. Q-device (P-device)
\end{abstract}

Keywords: semiconductor laser, DFB laser diode, optical logic device, optical computing

\section{INTRODUCCION}

Logic functions have been the cornerstone of a large number of systems and architectures, both in optical computing and in photonic switching. In most of the cases, these functions have been obtained either from specific devices with bistable characteristics or from hybrid configurations emulating this behaviour. Self-Electro optic Effect Devices (SEED) and etalon-like configurations have been extensively employed. Their main advantages are the possibility to obtain very fast switching times and their change in optical properties according to the employed wavelength. But these devices are sometimes difficult to find and their characteristics may greatly differ from one particular type to another. It is because that in this paper we present the possibility to obtain similar behaviour as the above mentioned specific devices, SEED or "on-off", with other devices commonly used in optical communications, namely, laser diodes. This line has been reported some years ago but the present intention is to present the real behaviour of these structures when a non-linear characteristic is need. The main objective of this paper is analyze the nonlinear properties of DFB laser amplifier and the way it can work as an elemental building block to construct Boolean Logic Functions as the authors of this paper have reported previously with conventional bistable devices.

We have been working on the design of optical structures to be applied as Boolean logic function for optical computing and optical switching and routing for optical communications. Our previous work was based on the definition of this logic structure using the methodology of simulation by functional model, and experimentally has been done with an optoelectronic approximation.

\section{BOOLEAN LOGIC GATE}

The optical programmable logic cell has been reported previously, a deeper description can be fond in [1]. Mainly it is consist in two devices. One is an On-Off device, denominated Q-device, and the other one is based in a SEED behavior, denominated P-device.

Q-device characteristics is easy and very well know, see Fig. 1, and allows just three types of digital function: AND, OR and ON. Figure 1 shows the ideal characteristic, in reality there is not an instantaneous transition between off and on respond, or between or and off. Time up and time down determine logic device time respond Time respond will affect mainly to bit rate processing and this is not the main objective to analyze in our work, even thinking we can not leave aside time study of logic gate. But first objective must be to be sure the device it will work and it is eficaz, in a second step, and depending on the application it will be study the way to improve consumes and time respond. 
Fig. 1.- Q-device block diagram and ideal input-output characteristic for the three control signals levels and the logic function to be performer

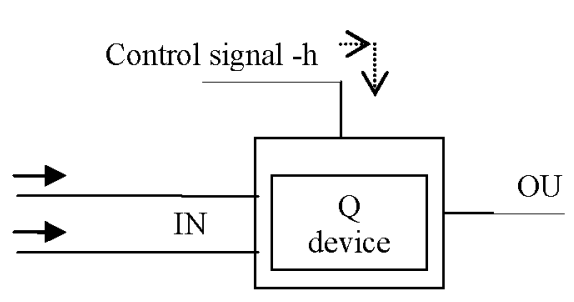

From the point of view of power characteristic, without bearing in mind times respond, some consideration must be look in detail. If bistability device are used, which is usually the case, exist a range where for the same input value, the output can take different values depending on the previous value of the input signal. Bistability device behaviour takes in account the history of the input signal. The consequences of this effect must be study in order to use the logic function as part of a more complicated structure. Beside the hysteresis cycle, tolerance of input digital signal and cw power level of control signal are very important. Some aspects of the impact of these have been presented on $[2,3]$.

Table I. Logic functions at the output of the logic gate

\begin{tabular}{ccccccc}
\hline \hline Input & \multicolumn{7}{c}{ Logic Function } \\
Signals & AND & OR & NAND & NOR & XOR & XNOR \\
$0 \quad 0$ & 0 & 0 & 1 & 1 & 0 & 1 \\
01 & 0 & 1 & 1 & 0 & 1 & 0 \\
11 & 1 & 1 & 0 & 0 & 0 & 1 \\
$\mathrm{I}_{1}+\mathrm{I}_{2}$ & $\mathrm{~g}_{3}$ & $\mathrm{~g}_{0}, \mathrm{~g}_{4}$ & $\mathrm{~g}_{1}$ & $\mathrm{~g}_{2}$ & --- & --- \\
$2 \cdot\left(\mathrm{I}_{1}+\mathrm{I}_{2}\right)$ & --- & $\mathrm{g}_{3}, \mathrm{~g}_{4}$ & --- & --- & $\mathrm{g}_{0}$ & $\mathrm{~g}_{1}, \mathrm{~g}_{2}$ \\
& \multicolumn{7}{c}{ Value of optical control signal } \\
\end{tabular}
behaviour and consequently it allows more Boolean logic function. Plus Qdevice function, AND \& OR, it is possible to implement NAND, NOR, XOR \& $\mathrm{XNOR}$. In Table $\mathrm{I}$ it is summary the Boolean functions for different control signal levels. In this way all the Boolean function can be done. The way to obtain a double value for input signals is resolved internally with the OPLC configuration or modifying on the device the level that must have the input to generate a change in the output, switching level. On table one it is also summary which function can be obtained with double power on a bit

"1", maintained the same power control signal levels. A better understanding on how can be change the control signal level is must be obtained directly with the switching power for transmission and reflection mode [1-3, 8]. This will lead to an specific shape of P-device characteristic show in fig. 5.

In next section we describe how we are using the laser diode in order to carry out the Optical Logic Cells (OLC).

\section{LOGIC GATE BY LASER DIODE BISTABILITY}

In the development of optical systems for ultra-high-speed signal processing and communications there have been several approaches, since the beginning of the eighties. One taken has been the mode locking of the semiconductors laser [4]. Another was the work on samplers, multiplexers and demultiplexers [5]. Also at that time there was a growing interest in the use of nonlinear properties of semiconductors for the design of optical elements to process optical pulses. Apply of nonlinear optical interaction as logic gate was implemented in a waveguide interferometer [6].

The components of an optical signal processing system should be compact, faster than room temperature electronics devices (i.e have a picosecond response time) and operate at the relative low power levels from semiconductor lasers (peak powers on the order of a few mWatts) Input and output pulses should be at the same optical frecuency. Also if the device development is toward the realization of an optical computer or an array of all-optical gates and switches, power and size become relevant.

In our work we do not have in mind any final complex architecture. We are evaluating different configuration of non-linearity that allows us to study a bistability device where the hysteresis cycle can be modify. We have chosen a semiconductor laser because of its clear advantage on size, power and cost. 
Much work has been performed on the optical bistability and switching in semiconductor lasers under intramodal light injection when the frecuency of injected light is in the vicinity of that of the slave laser. The laser denominated slave is the laser that acts us logic gate, and the injected light is the data signals to be processed. Other works use intermodal (sidemode) injection locking, the frecuency of the injected light is near a cavity mode which is different from that of the free-running slave laser [7].

Our work is based in intramodal light injection. First we have study a Fabry-Perot (FP) laser diode as an optical logic gate [8] on its different mode of operation, transmission and reflective. For Fabry-Perot structure rate equations have been analyzed the steady-state hysteresis, where the average internal optical power is plotted as a function of the incident power for different wavelengths. In this contribution with DFB laser diode we do not analyzed it.

In order to implement the Boolean logic function as described in previous section, the Q-device corresponds to the transmission mode of the laser diode represented on fig. 2(a). The more complex figure of P-device indicated in table I requires of a more complex configuration. It needs two laser diodes, one in reflective mode of operation and the other in transmission. In fig. 2(b) is represented as block diagram the LD structure.

(a)

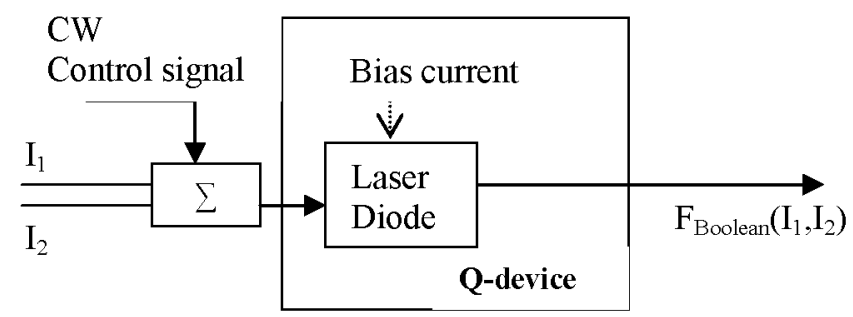

Figure 2.- Block diagram of (a) Qdevice and (b) P-device. $\sum$ - means adding optical signals by couplers.

(b)

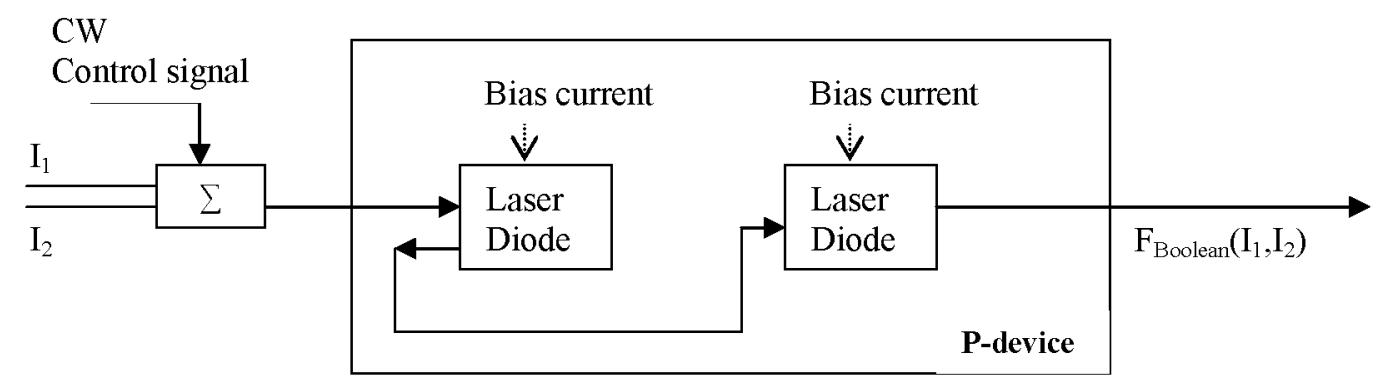

We obtain dispersive optical bistability in semiconductor laser operating below threshold current. But OB can be present also above threshold current. A deeper description on this can be found on [9].

\section{DFB LASER DIODE AS LOGIC DEVICE}

In a DFB- SLD [10] with transmission mode operation the dispersive optical bistability is present with a positive feedback loop. This occurs because there is an intensity-dependent refractive index in semiconductor laser and a cavity resonance. The intensity-dependent refractive index is due to the dependence of the refractive index on the carrier density. The feedback is provided by the built-in diffraction grating along the length of the active region. In reflective mode operation the hysteresis shape is different compared to the transmission mode, and both of then dependent on bias current and initial frecuency detuning. The bistability curves exhibit different switching threshold powers depending on the optical external signal wavelength. For transmission mode if the optical signal wavelength is father away from the cavity resonance, therefore it requires a larger incident power to seed the positive feedback loop.

The DFB laser diode bistability has been study and implemented in several occasions [11, 12, 13,14], but dynamical properties are not yet well defined. Depending on the applications OB must be obtained with bias over or below threshold current. For example, the switch-off time in a dispersive bistable laser amplifier is usually considered to be limited by the inverse of the effective carrier lifetime. A report [15] reveal that experimentally that the OB switch - off can be much faster than the carrier lifetime when the salve laser is biased above threshold. But this is not our goal.

In the present work we demonstrated that our configuration of the OLC allows to execute Boolean logic functions with maintained the same optical power level for a bit " 1 " and " 0 " on all the cases and it is only necessary to add a $\mathrm{CW}$ optical power to indicated to the device which Boolean function must be done. 


\section{SIMULATION RESULTS}

The viability of the configuration proposed as logic gate has been evaluated by VPIcomponentMaker ${ }^{\mathrm{TM}}$ Active Photonics. It is an integrated design environment for active photonic circuits and advanced semiconductor lasers. VPIcomponentMaker ${ }^{\mathrm{TM}}$ Active Photonics uses unique large-signal bidirectional-signal time-domain algorithms based on over 17 years research in time-domain laser modeling (the TLLM) [16]

\subsection{Q-device}

In this section we will describe briefly how is implemented the device. We have used two types of laser diode in order to obtain bistability: FP and DFB structure, but here only DFB structure is presented. Figure 1 shows the block diagram with 3 input signal, two for data and for a CW control signal that's allows the programmable of the logic function. Figure 3 is the model simulation and the transfer function. The optical data signals are obtained with external modulation. The random data is generated separately, sum and apply to an external modulator. Control signal is obtained by the same laser in order to maintain temporal coherence. The level of the control signal is changed by an variable attenuator.
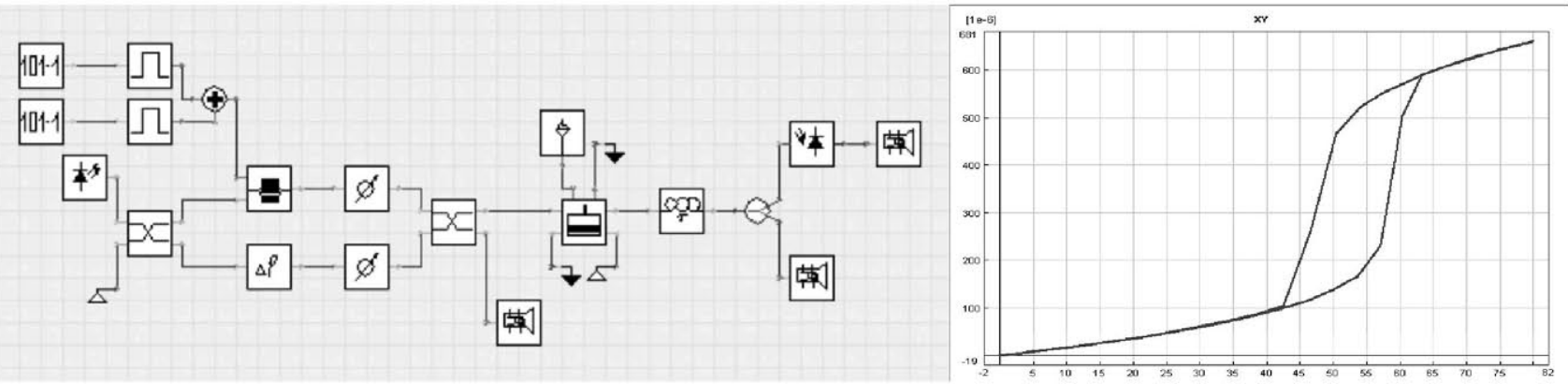

Figure 3.- Simulation model on VPIcomponentMakerTMActive Photonics. Q-device (DFB-SLD in transmission operation) output-input characteristic. Switching power $\approx 60 \mu \mathrm{W}$. On-off contrast $500 \mu \mathrm{W}$. Signal and resonance wavelength $\geq 1550 \mathrm{~nm}$

(a)

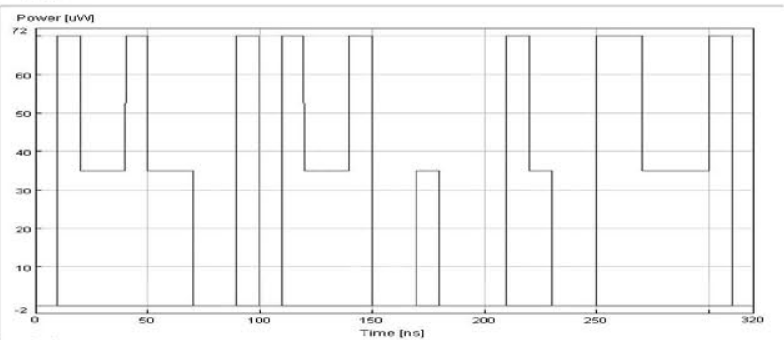

(c)

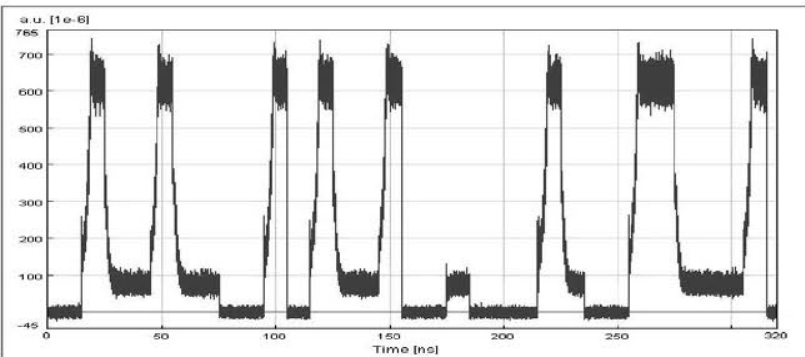

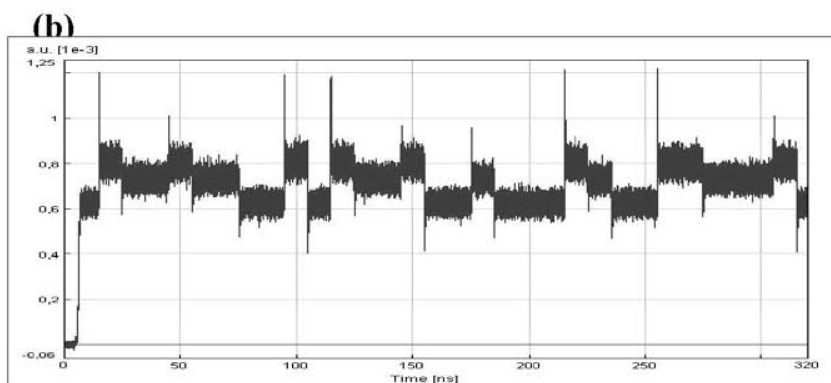

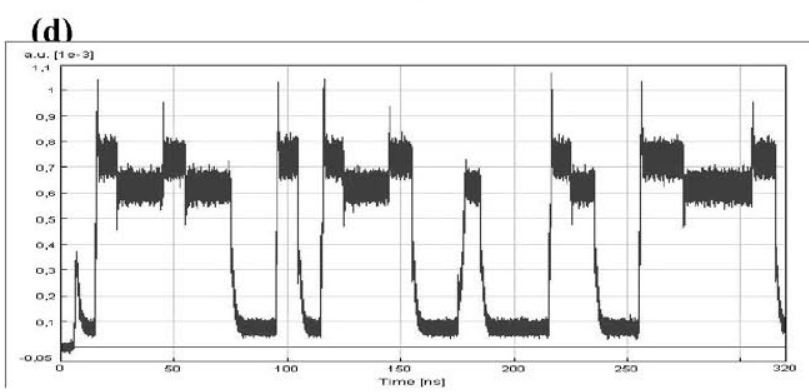

Figure 4.- Input $(35 \mu \mathrm{W})$ and output $(500 \mu \mathrm{W})$ data signals,

(a) Multilevel input data signal. Sum of random digital signals $I_{1}$ and $I_{2}$, both with same frecuency $100 \mathrm{Mb} / \mathrm{s}$.

(b) Output for control signal level $\mathrm{h}_{2}$ which correspond and $\mathrm{ON}$ - function with $\approx 0,5 \mathrm{~mW}$.

(c) Output for control signal level $\mathrm{h}_{0}(0 \mu \mathrm{W})$ which correspond and AND- function with a bit "1" $>500 \mu \mathrm{W}$

(d) Output for control signal level $\mathrm{h}_{1}(35 \mu \mathrm{W})$ which correspond and OR- function with a bit " $1 ">500 \mu \mathrm{W}$. 


\subsection{P-device}

The P-device was conceived as SEED device [17]. In order two obtain a similar transfer function the design structure consists on two coupled DFB semiconductor lasers; the first laser is operated in reflection, while the second is operated in transmission. The first laser performs the basic bulk of the logic processing, while the second acts as an optical power shareholding limiter. For a deeper description see [8]. Figure 5, 6 and 7 shows all the information about this device and their behavior when functions summary in table I are performed.

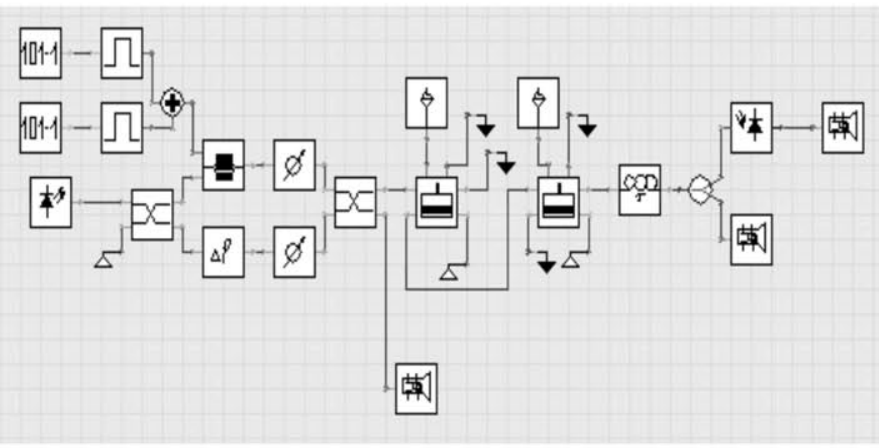

Figure 5.- Schematic model simulated by VPI_ComponentMaker ${ }^{\mathrm{TM}}$ software tool for P-device. Output-input characteristics show 3 switching power: off-on in $\approx 40 \mu \mathrm{W}$, on-off in $\approx 150 \mu \mathrm{W}$ and off-on in $\approx 300 \mu \mathrm{W}$. At least and output contrast of $\approx 200 \mu \mathrm{W}$.
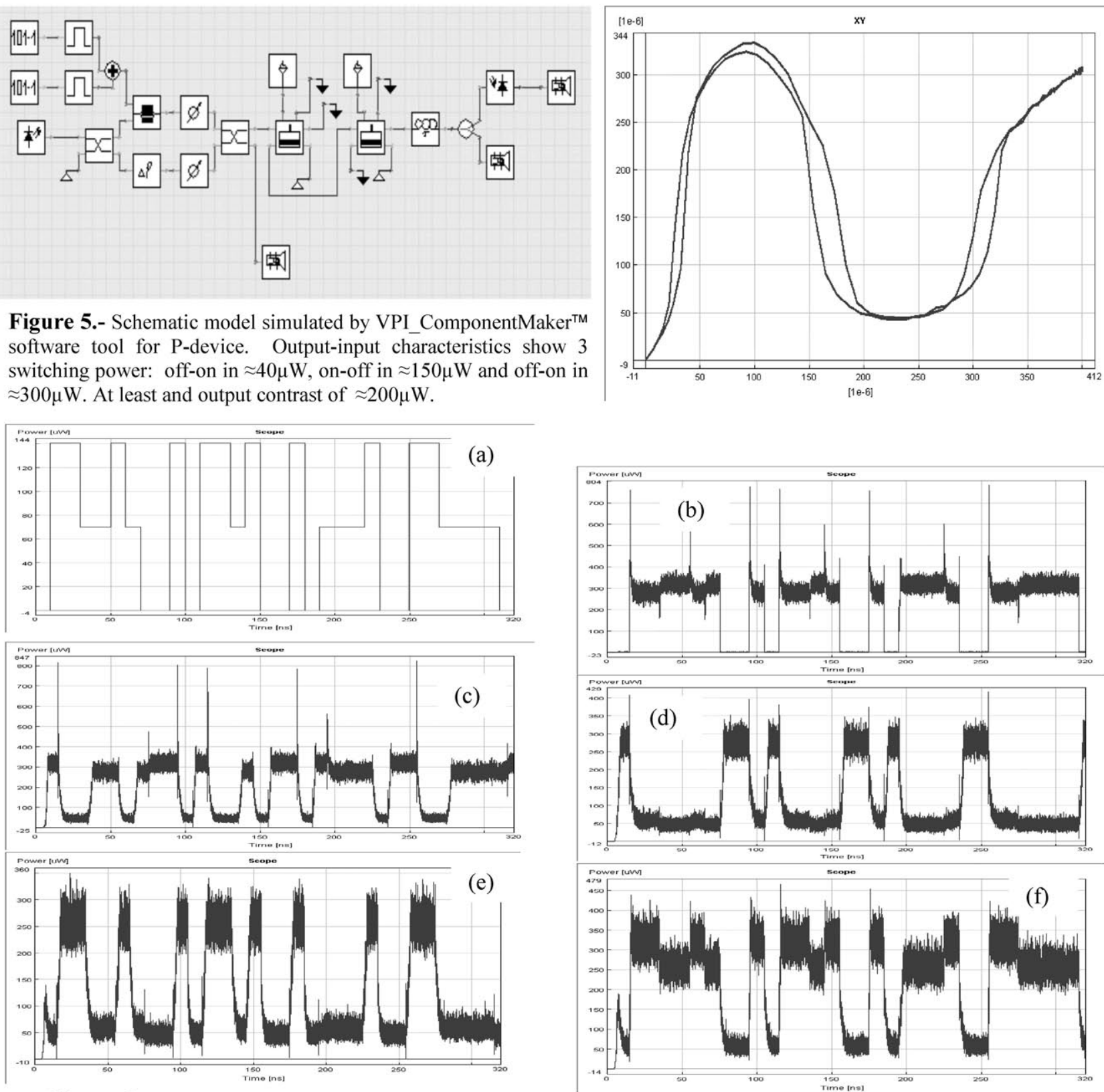

Figure 6.- Input (bit"1" $70 \mu \mathrm{W})$ and output $(>200 \mu \mathrm{W}$ ) data signals.

(a) Multilevel input data signal. Random digital signals $\mathrm{I}_{1}+\mathrm{I}_{2}$, both with same frecuency $100 \mathrm{Mb} / \mathrm{s}$.

(b) Output for control signal level $g_{0}$ which correspond and OR- function with $>200 \mu \mathrm{W}$.

(c) Output for control signal level $\mathrm{g}_{1}(\approx 70 \mu \mathrm{W})$ which correspond and NAND- function with a bit "1" $>200 \mu \mathrm{W}$.

(d) Output for control signal level $\mathrm{g}_{2}(\approx 140 \mu \mathrm{W})$ which correspond and NOR- function with a bit "1" $>200 \mu \mathrm{W}$.

(e) Output for control signal level $g_{3}(\approx 210 \mu \mathrm{W})$ which correspond and AND- function with a bit "1" $>200 \mu \mathrm{W}$.

(f) Output for control signal level $g_{4}(\approx 280 \mu \mathrm{W})$ which correspond and OR- function with $>200 \mu \mathrm{W}$. 
It is very well known that a logical function can be represented or done with different Boolean expressions. The basic ones are the functions AND and OR, that with an invert gate allows to define any logic purpose; we implement AND and OR just with the Q-device. NAND and NOR gates, which are more complex, required the P-device configuration. With this two, it is demonstrated, by De Morgan theorem, invert gate. Last function of Boolean algebra is the exclusive OR (XOR) which can be done with NAND and NOR gates. XOR function it has been since the 60's very important for data codification [6] and still used in communications applications [18]. In order to obtain this last function, the internal configuration of our OPLC [1] can be demonstrated applying double value to our input digital data. We simulated with same model of figure 5 with the input represented in figure 7 (b) and we obtain results of figure 7 (c)-(g).

As we can see there is an unavoidable feature of bistable action: the presence of an output intensity spike, as the phase must always past through a resonance during switch-up (and switch-down). This affect from a practical point of view mainly in timing as due to it there is an associated time delay before steady-state output is achieved. This spike can be seen in our simulation. In figure 7(c), when XOR is execute, we can see a higher spike when the transition from a zero to two " 1 " is being at the input data. A deeper approach study on noise and a way to choose the right configuration to execute Booleans function with laser diode can be found in [20].

On table II can be find the parameters apply to the slave laser diode on a Q-device and P-device on transmission mode. At it can be seen the polarization is under threshold, $0.88 \%$ of threshold current.

Table. II.- Laser Parameter for DFB -laser simulated

\begin{tabular}{lc}
\hline Laser Parameter & DFB \\
Cavity Length $(\mu \mathrm{m})$ & 300 \\
Left/Right Facet Reflectivity & - \\
Confinement Factor & 0.3 \\
Linear Material Gain Coeff. $\left(\mathrm{cm}^{2}\right)$ & $2.5 \cdot 10^{-16}$ \\
Linewidth Enhancement Factor & 5 \\
Linear Recombination Coeff. $(1 / \mathrm{s})$ & $1 \cdot 10^{8}$ \\
Bimolecular Recomb. Coeff. $\left(\mathrm{m}^{3} / \mathrm{s}\right)$ & $2 \cdot 10^{-16}$ \\
Auger Recombination Coeff. $\left(\mathrm{m}^{6} / \mathrm{s}\right)$ & $8.25 \cdot 10^{-41}$ \\
Transparency Carrier Density $\left(1 / \mathrm{m}^{3}\right)$ & $10^{24}$ \\
Fixed Internal Loss $(1 / \mathrm{m})$ & 1500 \\
Index Grating Coupling Coeff. $(1 / \mathrm{m})$ & 8000 \\
Bias current & $0.88 \cdot \mathrm{I}_{\text {th }}$ \\
Frequency detuning $(\mathrm{Ghz})$ & 14 \\
\hline
\end{tabular}

\section{CONCLUSIONS}

Any arithmetic-logic unit or an embebed digital design is mainly based on logic gates on its basic design before applying sequential logic. From this point of view it is very useful to have the same hardware to implement all the Boolean function used in those tasks. Here we have present how with one laser diode working under threshold current allow all the Boolean function. We demonstrate by a well know and probe simulation program of photonics device how we can done optical logic cell with distributed feedback semiconductor laser diode DFB-SLD. This type of configuration of laser diode allows a higher contrast, because of optical gain, and it is less noise compared to the FabryPerot laser diode.

The power of input digital data and control signal are on the order of hundred of $\mu \mathrm{W}$ which it is very reasonable level for a first approach to an optical digital architecture. The output power for a Q-device around half a miliwatt has allow the implementation of a P-device, and allows a fan-out of at least 6 on the lowest case. To maintain these levels of power it is essential the wavelength of data and control signal. It is desired that both type of signal maintain the same wavelength. In our simulation we have used the same laser as optical source for control and data signal.

A further study will be done related to data rate or processing rate. But this study depend mainly on the application of OLC; for communications employed in switching structures it is required a high velocity; for processing the interconnections of basic processing elements is even more important. 

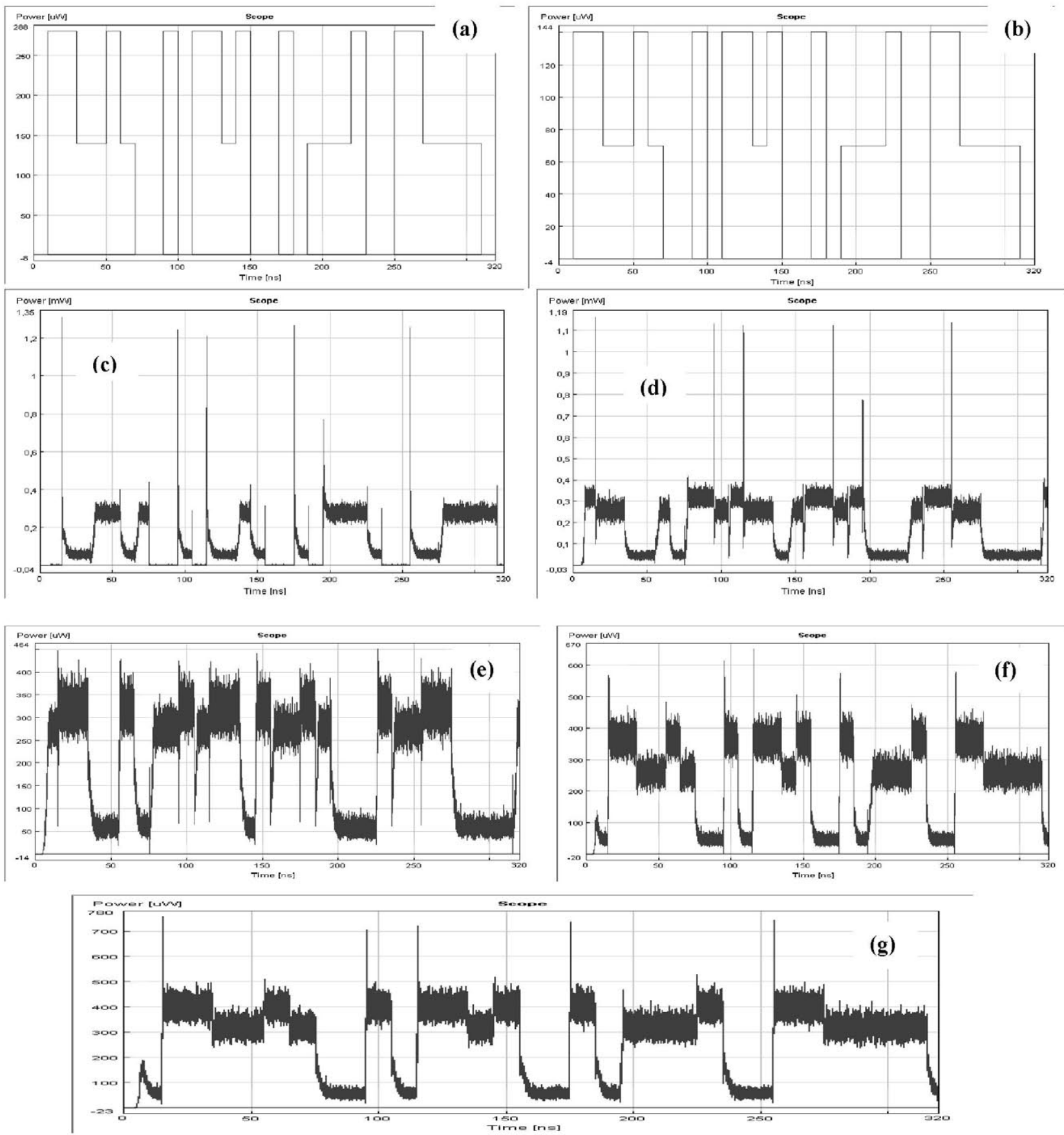

Figure 7.- Input (bit" $1 " 70 \mu \mathrm{W})$ and output $(>200 \mu \mathrm{W})$ data signals.

(a) Multilevel input data signal. Random digital signals $I_{1}+I_{2}$, both with same frecuency $100 \mathrm{Mb} / \mathrm{s}$, bit" 1 " $140 \mu \mathrm{W}$.

(b) Multilevel input data signal. Random digital signals $I_{1}+I_{2}$, both with same frecuency $100 \mathrm{Mb} / \mathrm{s}$, bit" 1 " $70 \mu \mathrm{W}$, same as Fig. 6(a)

(c) Output for control signal level $g_{0}$ which correspond and $\underline{\mathbf{X O R}}$ - function with $>200 \mu \mathrm{W}$.

(d) Output for control signal level $\mathrm{g}_{1}(\approx 70 \mu \mathrm{W})$ which correspond and XNOR-function with a bit "1" $>200 \mu \mathrm{W}$.

(e) Output for control signal level $\mathrm{g}_{2}(\approx 140 \mu \mathrm{W})$ which correspond and XNOR-function with a bit "1" $>200 \mu \mathrm{W}$.

(f) Output for control signal level $\mathrm{g}_{3}(\approx 210 \mu \mathrm{W})$ which correspond and OR- function with a bit " 1 " $>200 \mu \mathrm{W}$.

(g) Output for control signal level $\mathrm{g}_{4}(\approx 280 \mu \mathrm{W})$ which correspond and OR-function with $>200 \mu \mathrm{W}$. 


\section{ACKNOWLEDGMENTS}

This work was partly supported by CICYT, grant TIC2003-04309, and CAM "Comunidad Autónoma de Madrid", grant FPI- Formación de Personal Investigador.

\section{REFERENCES}

1. Gonzalez-Marcos, A.; Martin-Pereda, J. A.. "Digital chaotic output from an optically-processing element", in Optical Engineering, vol. 35, pp. 525-535, (1996).

2. Gonzalez-Marcos, A.; Martin-Pereda, J. A. "Analysis of irregular behavior on an optical computing logic cell", in Optics \& Laser Technology, vol. 32, No 6, pp. 457-466., (2000).

3. Gonzalez-Marcos, A.; Martin-Pereda, J. A. "Method to analyze the influence of hysteresis in optical arithmetic units", in Optical Engineering, vol. 40 No. 11, pp. 2371-2385. November 2001.

4. Haus, H.A. "Modelocking of semiconductor laser diodes" Japan. J. appl. Phhys., vol.20, pp. 1007-1020, June 1981.

5. Haus, H.A.; Kirsh, S.T. ; Mathyssek, K.; Leonberger, F.J. "Picosecond optical sampler" IEEE J. of Quantum Electron., vol. QE-16, pp.870 - 874, Aug. 1980

6. A.Lattes, H.A. Haus, F.J. Leonberger and E.P. Ippen "An Ultrafast all-optical gate" IEEE J. of Quantum Electron., vol. QE-19, pp.1718 - 1723, No. 11 Nov. 1983

7. Li, L. "Optical frecuency bistability and power bistability in semiconductors lasers" IEEE J. of Quantum Electron., vol. QE-31, pp.233 - 239, No. 2 Nov. 1995

8. Hurtado-Villavieja, A.; Gonzalez-Marcos, A. "An all-optical programmable logic gate with 1550nm laser diode amplifiers", Presented at Optics in Computing 2004, Engelberg (Switzerland), 21-23 April 2004.

9. Hui, R. "Static and Dynamical Properties of Dispersive Optical Bistability in semiconductor Lasers" J. of Lightwave Technology, vol.13, No.1, January 1995.

10. Otsuka, K.; Kobayashi, S. "Optical bistability and nonlinear resonance in a resonant-type semiconductor laser amplifier" Electro. Lett., Vol. 19, pp.262-263. 1983.

11. Maywar, D.N.; Agrawal, G.P., "Transfer-matrix analysis of optical bistability in DFB semiconductor laser amplifiers with nonuniform gratings" IEEE J. of Quantum Electron., vol. QE-33, pp.2029 - 2037, no. 11 Nov. 1997

12. Maywar, D.N.; Agrawal, G.P., "Effect of chirped gratings on reflective optical bistability in DFB semiconductor laser amplifiers" IEEE J. of Quantum Electron., vol. 34, pp.2364-2370, no.12, Dec. 1998

13. Kawaguchi, H.; Inoue, K.; Matsuoka, T.; Otsuka, K. "Bistable output characteristics in semiconductor laser injection locking" IEEE J. of Quantum Electron., vol. 21, pp.1314-1317, no. 9, Sep 1985

14. Nonaka, K.; Noguchi, Y.; Tsuda, H.; Kurokawa, T. "Digital Signal Regeneration with Side-Injection-LightControlled bistable Laser Diode as a Wavelength Converter", in IEEE Photonics Technology Letters, vol. 7, no. 1, January 1995.

15. Hui, R.; Paradisi, A.; Benedetto, S.; and Montrosset, I. "Fast optical switching of dispersive bistable laser diodes in the injection-locked regime" Lasers and Electro-Optics Society Annual Meeting, 1993. LEOS '93 Conference Proceedings. IEEE, pp. 518-519, 15-18 Nov. 1993

16. Lowery, A.J. "A two-port bilateral model for semiconductor lasers" IEEE J. of Quantum Electron., vol. QE28, pp.82-92, n. 1, Jan. 1992

17. Miller, D.A.B.; Chemla, D.S.; Damen, T.C.; Wood T.H.; Burrus, Jr C.A.; Gossar, A.C.; Wiegman, W. "The quantum well self-electrooptic effect device : optoelectronic bistability and oscillation, and self-linearized modulation" IEEE J. of Quantum Electron., vol. QE- 21, pp.1462 -1476, n.9, sept.. 1985

18. González-Marcos, A.; Martín-Pereda, J.A. "Digital Chaos Synchronization In Optical Networks". 'Optical Network Design and Modelling II'. Editores: G. de Marchis y R.Sabella. pp. 175-186. Kluwer Academic Publishers, 1999. ISBN: 0-412-84790-6

19. González-Marcos, A.; Martin-Pereda, J.A. "Photonic switching architectures with logic cells" En: SPIE ITCom2003. Active Optical Transmission Systems and Equipment for WDM Networking II Paper 5246.82. Orlando, Florida USA Sept. 05-09. 2003

20. González-Marcos, Ana P.; Martín-Pereda, José A.; Hurtado, A. "Bit noise from an optical logic gate with laser diodes" SPIE. Fluctuations and Noise in Photonics and Quantum Optics II, vol. 5468, pp. 56-65, (2004)

21. Sharfin, W. F.; Dagenais, M. 'High contrast, $1.3 \mu \mathrm{m}$ optical AND gate with gain', in Appl. Phys. Lett. 48, pp. 1510$1512,(1986)$. 